\title{
Visit-to-visit blood pressure variability and hip fracture: response to comments by Nagai et al.
}

\author{
T.-C. Li ${ }^{1,2} \cdot$ C.-C. $\operatorname{Lin}^{3,4,5}$
}

Received: 11 March 2019 / Accepted: 22 March 2019 / Published online: 2 April 2019

(C) International Osteoporosis Foundation and National Osteoporosis Foundation 2019

Dear editor,

We appreciate Dr. Nagai's valuable comment on our report. Many of these features of dementia such as fluctuations in cognition, disturbances in consciousness visual, and hallucinations may increase hip fracture risk [1]. Prior study has demonstrated that dementia is associated with increased hip fracture risk [2, 3]. In response to Dr. Nagai's letter, we examine the potential effect of dementia on the relationship between visit-to-visit blood pressure variability, measured by the coefficient of variation (CV) of and systolic blood pressure (SBP) and diastolic blood pressure (DBP), incident hip fracture in the elderly. In our dataset, a total of 49 elders with dementia at baseline with eight incident hip fracture cases during follow-up period resulted in a crude incidence rate of 25.37/1000 person-years, which is much higher than that of those without dementia (5.39/1000 person-years). We also found elders with dementia were associated with higher mean SBP-CV and DBP-CV, compared with those without dementia (SBP-CV and DBP-CV, $9.01 \pm 6.00$ and $11.16 \pm$ 7.75 for dementia vs. $7.47 \pm 5.61$ and $7.76 \pm 6.18$ for nondementia). To adjust for the confounding effect of dementia, we consider dementia in the final multivariate regression model and find the effect of SBP-CV remains the same: the

C.-C. Lin

cclin@mail.cmuh.org.tw

1 Department of Public Health, College of Public Health, China Medical University, Taichung, Taiwan

2 Department of Healthcare Administration, College of Medical and Health Science, Asia University, Taichung, Taiwan

3 School of Medicine, College of Medicine, China Medical University, 91 Hsueh-Shih Road, Taichung 40421, Taiwan

4 Department of Family Medicine, China Medical University Hospital, Taichung, Taiwan

5 Department of Medical Research, China Medical University Hospital, Taichung, Taiwan risk of hip fracture for SBP-CV per 5\% increase was significantly associated with a HR of $1.08(1.02,1.15)$. However, dementia is not a significant factor associated with an increased risk of hip fracture incidence (crude HR, 5.03, 2.51-10.09; adjusted 1.60,0.79-3.25), which may be due to its low prevalence). The other approach to rule the impact of dementia on the relationship between SBP-CV and hip fracture is to exclude those with dementia and we find SBP$\mathrm{CV}$ per 5\% increase exhibited similar significant HRs for the hip fracture of $1.08(1.01,1.15)$.

As population is fast ageing, the prevalence of dementia would increase and dementia may become an important factor that should be considered for hip fracture incidence. The authors would like to thank Dr. Nagai for the judicious reading of our manuscript and valuable comments which greatly inspired us to further explore the relationship between blood pressure variation and hip fracture and provide new insights into the field of hip fracture.

\section{Compliance with ethical standards}

Conflicts of interest None.

\section{References}

1. Friedman SM, Menzies IB, Bukata SV, Mendelson DA, Kates SL (2010) Dementia and hip fractures: development of a pathogenic framework for understanding and studying risk. Geriatr Orthop Surg Rehabil 1:52-62

2. Bohlken J, Jacob L, Schaum P, Rapp MA, Kostev K (2017) Hip fracture risk in patients with dementia in German primary care practices. Dementia (London, England) 16:853-864

3. Wang HK, Hung CM, Lin SH, Tai YC, Lu K, Liliang PC, Lin CW, Lee YC, Fang PH, Chang LC, Li YC (2014) Increased risk of hip fractures in patients with dementia: a nationwide population-based study. BMC Neurol 14:175

Publisher's note Springer Nature remains neutral with regard to jurisdictional claims in published maps and institutional affiliations. 